\title{
Las variables no son pizzas: el milagro es la multiplicación de los panes, no la multiplicación de las sub hipótesis
}

\author{
The variables don't are pizzas. The miracle is the multiplication \\ of bread don't multiplication of sub-hypotheses
}

Francis Díaz Flores ${ }^{1}$, (iD Fernando Pino Apablaza ${ }^{2} \quad$ (D) Carmen Jackeline Montenegro Vigo ${ }^{1}$

Elías Jesús Mejía Mejía ${ }^{1}$

${ }^{1}$ Universidad Nacional Mayor de San Marcos. Lima, Perú

${ }^{2}$ Universidad Le Cordon Bleu. Lima, Perú

Recibido: 12/03/2021 Revisado: 18/04/2021_ Aceptado: 08/05/2021Ｐublicado: 31/06/2021

\section{RESUMEN}

Este artículo explica por qué no es pertinente formular sub hipótesis, cuando en una investigación explicativa o una investigación correlacional no causal, se trabaja con dos variables, a propósito que la gran mayoría de estudiantes de posgrado de las universidades del Perú lo están haciendo, como práctica generalizada, en sus tesis, y cuyo origen es desconocido. Estos investigadores dividen en sus dimensiones las variables independientes $\mathrm{y}$, a partir de cada una de ellas, formulan las sub hipótesis. Algunos investigadores formulan sub hipótesis descomponiendo la variable independiente y la variable dependiente en sus respectivas dimensiones. Otros, descomponen la variable dependiente en sus dimensiones para formular sub hipótesis. Las sub hipótesis suelen plantearse cuando en las investigaciones multivariadas o factoriales, se trabaja con más de una variable independiente, es posible formular sub hipótesis a partir de cada una de las variables independientes. En este caso, la formulación de sub hipótesis se hace cuando la estrategia de prueba de hipótesis es el Análisis de Varianza. Palabras clave: Hipótesis, sub hipótesis, problemas, problemas específicos, dimensiones, análisis de varianza, regresión múltiple.

\begin{abstract}
This article explains why it is irrelevant to formulate sub-hypotheses, when in an explanatory research or non-causal correlation research, two variables are worked on, with the aim that the vast majority of graduate students of the universities of Peru are doing so, as a general practice, in their thesis, and whose origin is unknown. These researchers divide the independent variables into their dimensions and, from each of them, formulate the sub-hypotheses. Some researchers formulate sub hypotheses by decomposing the independent variable and dependent variable into their respective dimensions. Others break down the dependent variable into its dimensions to formulate sub-hypotheses. Sub-hypotheses are often raised when in multivaria-
\end{abstract}


te or factorial investigations, more than one independent variable is worked on, it is possible to formulate sub-hypotheses from each of the independent variables. In this case, the formulation of sub-hypotheses is done when the hypothesis test strategy is the Variance Analysis. Keywords: Hypotheses, sub-hypotheses, problems, specifics problems, dimensions, analyze of variance, multiple regression.

\section{INTRODUCCIÓN}

Este artículo explica por qué no es correcto formular sub hipótesis, cuando en una investigación explicativa o una investigación correlacional no causal, se trabaja con dos variables. Sin embargo, la mayoría de las tesis de posgrado lo están haciendo. Estos investigadores dividen en sus dimensiones las variables independientes $\mathrm{y}$, a partir de cada una de las dimensiones formulan las sub hipótesis. Algunos investigadores formulan sub hipótesis descomponiendo la variable independiente y la variable dependiente en sus respectivas dimensiones. Otros, descomponen la variable dependiente en sus dimensiones para formular las sub hipótesis. Las sub hipótesis suelen plantearse cuando en la investigación multivariada o factorial, se trabaja con más de una variable independiente. Pero, aún en este caso, la formulación de sub hipótesis se hace cuando la estrategia de prueba de hipótesis es el Análisis de Varianza. Cuando la estrategia de prueba de hipótesis es la Regresión Múltiple, no se puede formular sub hipótesis pues las variables independientes están en covarianza entre ellas y por eso, al contrario del caso anterior, sólo se formula una hipótesis general para estudiar con ella el efecto concurrente y concomitante de las variables independientes en la dependiente.

En la mayoría de las tesis de maestría y doctorado, los graduandos plantean, no solo problemas, sino problemas específicos, elaboran una matriz de problematización, un árbol de pro- blemas que, algunas veces, grafican con una espina de pescado. Hemos revisado cuidadosamente las obras de Popper, (2017), Bunge, (1983), Kerlinger y Lee, (2002), Klimovsky, (1994) Hernández, Fernándezy Baptista(2009), por tratarse de autores reconocidos como autoridades en la materia y, en ninguna de ellas, encontramos alusiones a las sub hipótesis.

Estos investigadores plantean, además, problemas generales y problemas específicos, redactan objetivos generales $y$, objetivos específicos y formulan hipótesis generales e hipótesis específicas. En el siguiente enlace electrónico se encuentra este tipo de trabajos:

https://cybertesis.unmsm.edu.pe/bitstream/handle/20.500.12672/6662/Mu\%c3\%b1oz_am.pdf?sequence $=1 \&$ is Allowed $=\mathrm{y}$.

El siguiente enlace tiene más información: https://cybertesis.unmsm.edu.pe/ bitstream/handle/20.500.12672/4925/ Cuba_rl.pdf?sequence $=1 \&$ isAllowed $=\mathrm{y}$. En este otro enlace se halla más información al respecto:

https://cybertesis.unmsm.edu.pe/bitstream/handle/20.500.12672/429/Acu\%c3\%bla_op.pdf?sequence $=1 \&$ isAllowe$\mathrm{d}=\mathrm{y}$.

Hay otros en cybertesis, como este último:

https://cybertesis.unmsm.edu.pe/bitstream/handle/20.500.12672/4485/Farfan_tc.pdf?sequence $=1 \&$ isAllowed $=y$ 
Cuando los graduandos trabajan con dos variables, una independiente y otra dependiente, no es posible formular sub hipótesis ni mucho menos plantear problemas específicos. Esto se hace cuando el investigador trabaja con dos o más variables independientes y una dependiente, situación en la que sí es posible formular sub hipótesis, a partir de cada una de las variables independientes, pero en algunos casos.

Este análisis genera el título del presente artículo porque los investigadores, al trabajar tan sólo con una variable independiente, para obtener las sub hipótesis, la dividen en tantas partes como dimensiones hayan identificado cuando deseaban operacionalizarla. Según esta lógica, plantean el problema general y los problemas específicos y el objetivo general y los objetivos específicos y, naturalmente, la hipótesis general y las sub hipótesis. El objetivo de toda investigación es demostrar la hipótesis, de modo que los objetivos podrán quedar implícitos. Sin embargo, los graduandos redactan objetivos generales y objetivos específicos en función de cada una de las dimensiones identificadas de la variable independiente. Otros graduandos dividen la variable dependiente en sus dimensiones constitutivas y así tejen, a partir de estas divisiones, una maraña de absurdos. Toda hipótesis se redacta en forma literal, pero para probarla con métodos estadísticos, debe ser formalizada en términos de una función matemática, disciplina que reconoce al símbolo X como la variable independiente $\mathrm{y}$, al símbolo Y, como la dependiente. En este sentido, la función es la siguiente: $\mathrm{Y}=\mathrm{f}(\mathrm{X})$. Es inusual formular dicha función del siguiente modo: $\mathrm{X}=\mathrm{f}(\mathrm{Y})$. En este caso, se está alterando la formulación matemática al asignar, arbitrariamente, la función de variable independiente a
Y, y la función de variable dependiente a X. Otra situación observada es que los graduandos redactan el mismo número de conclusiones y el mismo número de recomendaciones, según el número de sub hipótesis formuladas, situación que no siempre es aceptable.

\section{Clasificación, caracterización o tipificación de una investigación}

Para realizar una investigación es necesario conocer, con la mayor profundidad posible, su naturaleza y, una manera eficaz de hacerlo, es clasificarlas, tipificarlas o caracterizarlas, según criterios pertinentes. La literatura revisada da cuenta de diferentes clasificaciones de las investigaciones, pero sin precisar los criterios de clasificación de los que han partido. Toda clasificación, que se caracteriza por ser exhaustiva y excluyente, debe especificar el criterio según el cual se ha hecho la clasificación.

Según el principio de la exhaustividad, toda investigación que se clasifica debe, necesariamente, estar incluida en alguna clasificación del criterio asumido. No es posible que, planteado el criterio, una investigación no sea incluida en ninguna clasificación establecida por el criterio. Según el principio de la exclusión, una investigación, que ya fue incluida en una clasificación del criterio, ya no puede aparecer en otra clasificación del mismo criterio. Un ejemplo de este caso es el título de una telenovela que hace años tuvo mucho éxito entre las amas de casa: La fea más bella. Los adjetivos bella y fea corresponden a la clasificación del criterio belleza. De modo que no es correcto incluirla dos veces en la clasificación del mismo criterio. En cambio, se puede decir la fea más flaca o la bella más gorda, pues flaca y gorda corresponden a otro criterio, el criterio peso. 
Las investigaciones son diferentes unas de otras, no todas son iguales, y por esto es preciso conocerlas mediante un conjunto de criterios pertinentes de clasificación, algunos de los cuales son los siguientes, según Mejía (Mejía, 2017).

Según el tipo de conocimientos previos. Según este criterio, las investigaciones son filosóficas y científicas. Como los graduandos de las diversas escuelas de posgrado cursan maestrías o doctorados vinculados con las ciencias, la mayoría de las investigaciones o tesis que sustentan son científicas. Las investigaciones filosóficas también se aceptan para otorgar un grado de magíster o doctor. En este caso, el graduando reflexiona sobre la ciencia o los resultados de su investigación.

\section{Según la naturaleza del objeto de estudio} de las investigaciones científicas. Según este criterio, las investigaciones científicas son formales $\mathbf{y}$ factuales. Los graduandos que cursan maestrías o doctorados en matemática o lógica, por la naturaleza de las disciplinas que cultivan, realizan investigaciones formales demostrando, con métodos específicos, las formas del pensamiento. Los graduandos que cursan maestrías o doctorados relacionados con las ciencias factuales, es decir, las que se ocupan de los hechos o de los fenómenos empíricos, realizan investigaciones factuales. La naturaleza de la tesis depende de la naturaleza de los estudios de posgrado que hayan cursado los graduandos.

\section{Según el objeto de estudio de las ciencias fac-} tuales. Según este criterio, las investigaciones son naturales y sociales. Cada graduando, según los estudios que haya realizado, o según el grado a optar, debe realizar su investigación en el campo de las ciencias naturales, llama- das las ciencias duras, como la física, la química o la biología, o en las ciencias sociales, llamadas ciencias blandas, como la educación, la administración, la economía, la psicología, la sociología y un número, no finito, de ciencias sociales que surgen conforme avanza la investigación del comportamiento humano.

Según la pregunta que se plantea en el problema. Según este criterio, las investigaciones científicas son teóricas y prácticas. Las investigaciones teóricas, a su vez, son de tres tipos: descriptivas, explicativas y retrodictivas. Las investigaciones descriptivas se subdividen, a su vez, en dos tipos: las predicativas no causales y las relacionales no causales. Algunos especialistas sostienen que este tipo de investigaciones se puede hacer sin formular hipótesis, punto de vista que no compartimos.

Otra es la situación cuando el investigador desea saber las causas de un determinado fenómeno y se pregunta ¿por qué X es cómo es? Esto ya es una pregunta de mayor profundidad, pues se trata de conocer la causa o las causas del efecto que se estudia. Se trata de las investigaciones explicativas y, en este caso, es inconcebible no proponer hipótesis que traten de explicar la relación planteada entre $X y Y$. Las investigaciones explicativas constituyen el ideal científico. Las causas pueden ser una o varias, y según esto, las investigaciones son bivariadas, de dos variables, una causa que produce un efecto, como el calor dilata los metales o multivariadas o factoriales, en las que se hipotetiza que más de una causa produce el efecto deseado, como por ejemplo la actitud de muchas personas ante las vacuna anti COVID-19, que deciden no aceptar la vacuna, ante tantas teorías conspirativas que se van difundiendo en las redes sociales y que se conocen como fake news. Estas teorías conspi- 
rativas son muchas, por ejemplo, que el virus ha sido creado en un laboratorio chino, que Bill Gates y Joseph Maiman han financiado la pandemia, que la humanidad está sufriendo por sus pecados, que la vacuna instala un chip en el cuerpo del paciente para monitorearlo o que la vacuna modifica el ADN de las personas. (BBC News/Mundo, 2021). Estas son las posibles causas de su actitud para no vacunarse, a las que denominamos variables independientes. Hasta diciembre de 2020, el porcentaje de personas con actitud contraria a la vacuna era de aproximadamente de $50 \%$ de la población. En los primeros meses del 2021, cuando se ha vacunado a quienes estaban en la primera línea de la lucha contra el COVID-19, este porcentaje va disminuyendo progresivamente. La ciencia explica que el COVID-19, se previene con la vacuna, siempre y cuando se alcance la 'inmunidad de rebaño', es decir, que toda la humanidad esté vacunada, porque sólo así se evitarán los contagios. También la ciencia advierte que la vacuna sólo evita la mortalidad y la hospitalización, por lo que las personas vacunadas deben seguir observando los protocolos de bioseguridad.

Pero ha sucedido que los investigadores de la Universidad Peruana Cayetano Heredia y de la Universidad Nacional Mayor de San Marcos, han actuado en términos de 'sálvese quien pueda'. Así, un conjunto de funcionarios administrativos, familiares y allegados, han sido vacunados en secreto, lo que ha destruido las carreras profesionales de reconocidos investigadores, según el Informe de la Comisión Sectorial Investigadora de la Aplicación de la Vacuna Candidata Contra la COVID-19, constituida por Resolución Ministerial $\mathrm{N}^{\circ}$ 225-2021-MINSA que presidió el Dr. Fernando Carbone. (Informe de la Comisión Secto- rial Investigadora de la Aplicación de la Vacuna Candidata Contra la COVID-19, 2021).

Las investigaciones retrodictivas, tratan de describir o explicar el pasado. Retroceden hacia el pasado. Estas investigaciones pretenden conocer cómo fueron las cosas, lo que sería la descripción del pasado o, por qué las cosas en el pasado fueron como fueron, que sería la explicación del pasado. Lamentablemente, muy pocos graduandos de posgrado acometen este tipo de investigación, pese a su importancia para la ciencia, pues se trata del conocimiento del pasado, de la historia de la ciencia, que permite conocer y explicar mejor el presente.

Otro tipo de investigaciones que corresponde al criterio que se analiza, son las investigaciones prácticas. Estas investigaciones responden a la pregunta ¿qué hacer para que la situación A, supuestamente indeseada, se convierta en $B$ ?, la situación deseada. Un ejemplo actual referido a la sociedad peruana es que ya se sabe, por descripción, que los niveles de corrupción han llegado a extremos muy elevados, situación A. Entonces, qué se debe hacer para bajar estos altos niveles de corrupción en la sociedad peruana, situación B. Otro caso es el de la educación superior, cuya calidad es muy baja, situación $A$, y se debe hacer algo para que sus niveles de calidad se incrementen, situación B. Precisamente la Ley Universitaria 30220 se fundamenta en la necesidad de mejorar la calidad de la educación superior, se crea la Superintendencia Nacional de la Educación Superior Universitaria, SUNEDU, y se adscribe la Universidad Peruana al Ministerio de Educación en el supuesto que, con estas medidas, la calidad de la educación superior universitaria mejorará significativamente. Sin embargo, en los países desarrollados, la cali- 
dad de la educación es alta y no existe en ellos una Superintendencia de Educación Superior y, en los Estados Unidos de Norteamérica, por ejemplo, no existe un Ministerio de Educación. Sin embargo, tres universidades norteamericanas encabezan el Ranking Mundial de Universidades 2021 de la Quacquarelli Symonds, (2021): $1^{\circ}$, el Instituto Tecnológico de Massachussets, $2^{\circ}$, la Universidad de Stanford, y, $3^{\circ}$, la Universidad de Harvard. Las investigaciones de este tipo también reciben el nombre de investigaciones tecnológicas o aplicadas.

Otro caso es que, en la Universidad Peruana Cayetano Heredia, que ocupa el tercer lugar en el Ranking de universidades del Perú, según QS, (Quacquarelly Symonds, 2021), protagoniza el escándalo del "Vacunagate", como lo señala Lucero Ascarza en el artículo periodístico en Salud con Lupa del 04.03.2021, (Ascarza, 2021), asuntos que proporcionan razones para afirmar que la conducta humana es elusiva e impredecible.

En la reciente investigación sobre la vacuna contra el COVID-19, la investigación es de este tipo, pues no se trata de encontrar conocimiento acerca del virus, sino evitar los contagios. Con la investigación para producir la vacuna, se trata de lograr la situación $B$, la deseada, es decir inmunizar a la población para reducir las alarmantes tasas de mortalidad. Esta es una típica investigación aplicada. Sin embargo, muchos graduandos de posgrado no la acometen, pese a que este tipo de investigaciones son muy útiles en el avance de la ciencia. Estas investigaciones comienzan con el conocimiento teórico de una realidad, de la explicación científica, la dimensión más importante de la ciencia.

Según el método de contrastación de la hipótesis. Según este criterio, las investigaciones son de causa a efecto y de efecto a causa, que también llama investigación ex post facto. La secuencia del método en la investigación es muy importante, por eso se distinguen investigaciones que van de la causa al efecto, que es posible cuando la variable independiente es de naturaleza activa, es manipulable. En caso contrario, si la variable independiente es atributiva, no es posible la manipulación y este tipo de investigación no se puede realizar. En este caso es muy importante el criterio de clasificar las variables por su naturaleza.

Cuando el método discurre de la causa al afecto, las investigaciones pueden subdividirse en tres tipos: pre experimentales, experimentales y casi experimentales. Las investigaciones pre experimentales son las que no controlan las variables intervinientes y, por tanto, los resultados que producen son poco confiables, por lo que no es recomendable realizar investigaciones pre experimentales para optar un posgrado. El segundo tipo lo constituyen las investigaciones experimentales, en las que sí es posible controlar las variables intervinientes. La experimentación se produce cuando se cumplen las siguientes condiciones: a) se investiga con dos grupos, por lo menos, b) los grupos son iguales, c) el mismo investigador forma los grupos y, d) el sujeto que participa en la investigación no debe saber si pertenece al grupo experimental o al grupo de control. Esta es una estrategia muy completa y es la que se aplica en las ciencias duras.

Últimamente, por los ensayos clínicos que se hacen con respecto a las vacunas anti COVID-19, se sabe que las investigaciones experimentales se realizan por etapas o fases. Previamente se experimenta con animales, luego con seres humanos voluntarios en una o varias fases, según la rigurosidad del ex- 
perimento. Sólo después de culminada satisfactoriamente la experimentación y luego de satisfacer los protocolos de las autoridades de salud de un país, se aplica la vacuna a la población en general. La vacunación masiva, ya no es experimento, sino la aplicación del remedio descubierto en base a la evidencia obtenida en las fases experimentales.

El problema radica en que, en las ciencias blandas, no es posible cumplir con las condiciones del experimento científico, por lo que se ha acuñado el término cuasi experimental, para aludir a la situación en que si se pretende hacer una investigación experimental y no se puede cumplir alguna de las condiciones o sólo se cumple algunas. Este tipo de investigación es la que realiza la gran mayoría de los graduados de posgrado, pues muchos de ellos cursan estudios en las áreas de las ciencias sociales, en las que, como se ha dicho, no es posible hacer experimentación y sólo se puede hacer cuasi experimentación.

Otro escenario es el conformado por el discurrir del método en sentido contrario, del efecto a la causa. En este caso se trata de la investigación ex post facto, que muy pocos graduandos realizan pese a la alta versatilidad que ofrece para realizarla. En este caso, el investigador ya está ante los efectos y no tiene identificadas las causas que han producido el efecto. En la investigación del COVID-19, se está trabajando en este sentido, pues el COVID, 19 apareció de pronto, se produjo el efecto, se declaró la pandemia, y la investigación, en estos últimos meses, radica en identificar las causas del COVID-19, las que aún no han sido identificadas por completo.

Según el método de estudio de las variables. Según este criterio, las investigaciones son cuantitativas, cualitativas y mixtas, que algunos autores las llaman cualicuantitativas. Para saber si una investigación es cuantitativa se puede preguntar si entre dos personas, una es más alta que la otra. Si se acepta que es correcto hablar en estos términos, se asume que la investigación es cuantitativa, pues la talla de las aludidas personas es una cantidad que se puede medir porque tiene masa, es una magnitud. Muchas veces, es correcto hablar en estos términos, pero no existen instrumentos de medición. En este caso, se debe elaborar el instrumento o la escala para medir la variable, siguiendo el principio establecido por Galileo: "Mide lo que se puede medir; y lo que no, hazlo medible." (Muy Interesante, 2021). Cuando no es posible hablar en términos de más o menos, se asume que las investigaciones son cualitativas, como el estado civil, pues no se puede decir que Juan es más casado que Pedro. En los últimos años, en que emerge el paradigma hermenéutico, se hacen investigaciones cuantitativas y luego se analizan e interpretan los datos, investigación cualitativa, con lo que se terminan haciendo investigación mixta.

Según el número de variables. Según este criterio, las investigaciones científicas son univariadas, bivariadas y multivariadas, llamadas también factoriales. Las investigaciones descriptivas predicativas no causales son univariadas pues sólo estudian un fenómeno y, por tanto, tienen una sola variable. Algunos especialistas las llaman investigaciones propositivas. Las bivariadas, establecen la relación de causa a efecto entre dos variables, como cuando se dice: el calor dilata los metales. Las investigaciones son multivariadas, cuando se trabaja con más de una variable independiente, que actúan sobre la dependiente, como en la siguiente hipótesis: La motivación de logro, aptitudes intelectua- 
les, calidad del desempeño docente, utilización de métodos didácticos de aprendizaje y el apoyo familiar, son factores que influyen en el aprendizaje de los estudiantes. En las ciencias sociales que estudian la conducta humana, fenómeno elusivo e impredecible, no se puede asilar un solo factor causal de la variable dependiente, por lo que se asume que la concurrencia y hasta concomitancia de factores produce el efecto que se estudia.

Según el ambiente en el que se realizan. Según este criterio, las investigaciones son bibliográficas, de laboratorio y de campo. Las bibliográficas, conocidas también como de gabinete, son las que cuando un historiador, desde su gabinete de trabajo y, a partir de fuentes históricas que dispone, escribe la historia. Las investigaciones son de laboratorio, cuando los investigadores de las ciencias duras realizan experimentos científicos estrictos, precisamente en sus laboratorios en los que es posible controlar todas las variables intervinientes. Las investigaciones de campo, son las que realizan los arqueólogos cuando realizan excavaciones, los sociólogos, cuando estudian el comportamiento de la sociedad, los contadores cuando analizan documentos contables o los educadores cuando estudian el desempeño de los estudiantes en el aula, que es el campo, lugar o escenario donde se realizan los procesos educativos.

Según la prelación de los datos que producen. Según este criterio, las investigaciones son primarias y secundarias. Las investigaciones son primarias porque el investigador reporta hallazgos de primera fuente, como el arqueólogo que descubre la tumba de Tutankamón o los investigadores del coronavirus que aún no informan el resultado de la investigación que están realizando acerca de la naturaleza y origen del virus. Estos investigadores, cuando logren descubrir la naturaleza del virus, a nuestro juicio, ganarían el Premio Nobel de Medicina. Estas investigaciones primarias, por su importancia para la ciencia, se denominan también traslacionales, pues trasladar los resultados de sus hallazgos hacia otras ciencias o disciplinas que hacen investigación secundaria, a partir de las fuentes primarias reportadas por las investigaciones traslacionales. En este sentido, las investigaciones secundarias se nutren del conocimiento producido por las traslacionales, como la historia, que se nutre de la arqueología, la educación que se nutre de la psicología o la economía que se nutre de la estadística.

Según las disciplinas que participan. Según este criterio, las investigaciones son unidisciplinarias, interdisciplinarias, multidisciplinarias, transdisciplinarias, pluridisciplinarias y de disciplinariedad cruzada. El avance científico está haciendo desaparecer las fronteras decimonónicas de la ciencia y somos testigos que están emergiendo nuevos paradigmas epistemológicos que explican que no es posible seguir investigando unidisciplinarmente. (Piaget, 1979). De ahí que la investigación se está realizando en base a equipos o grupos de investigación, conformados por especialistas de diferentes disciplinas que, en conjunto y colaborativamente, procuran obtener conocimiento de la realidad natural o social, que se presenta en forma integrada.

\section{Clasificación de las variables}

Otro asunto que pasan por alto los graduandos de posgrado al hacer sus tesis, es una precisa identificación y clasificación de las variables que estudian. Una eficaz clasificación de las 
variables, a partir de criterios pertinentes, permite conocer, en forma precisa, las variables de estudio para caracterizarlas adecuadamente, operacionalizarlas en los mejores términos y elaborar los instrumentos de recolección de datos más apropiados para estudiarlas. Mejía explica los siguientes criterios. (Mejía, 2017).

Por la función que cumplen en la hipótesis. Según este criterio, las variables son independientes, dependientes e intervinientes. Una variable es independiente no por su naturaleza sino por la función de supuesta causa que el investigador le asigna al formular la hipótesis. Por ejemplo, el entrenamiento integral, no es siempre una variable independiente; en otras hipótesis, puede funcionar como variable dependiente. La condición de ser independiente o dependiente, no depende de las variables, sino de la decisión que el investigador adopta al asignarle la función de supuesta causa o posible efecto. En la siguiente hipótesis: el entrenamiento integral es un factor que contribuye a mejorar la calidad del servicio educativo, la variable entrenamiento integral ha recibido la función de actuar como supuesta causa. En cambio, en la siguiente hipótesis: la motivación de los docentes por mejorar la calidad de su práctica pedagógica, constituye un factor que contribuye a fortalecer su entrenamiento integral, la variable entrenamiento integral ha recibido la función de posible efecto.

Las variables intervinientes, si no son advertidas o detectadas oportunamente, pueden actuar como posibles causas y afectar los valores de las variables dependientes y así, pueden producir, lo que Kerlinger y Lee denominan, resultados espurios de la investigación. (Kerlinger y Lee, 2002). Una buena estrategia de investigación, que muchos autores inco- rrectamente denominan diseño, puede servir para detectar a tiempo la actuación de las variables intervinientes. Pero si el investigador ya las detectó, en virtud de la calidad de su estrategia, ya no son variables intervinientes.

Por su naturaleza. Según este criterio, las variables son atributivas y activas. En el ejemplo que estamos explicando, el entrenamiento integral es variable atributiva, pues es un conjunto de competencias que el docente ha adquirido en sus procesos de capacitación. Esta variable ya se ha constituido en parte de su persona, de su ser esencial y es como su nombre, su género o su edad. Por esta razón, no es manipulable y sólo puede ser analizada mediante instrumentos de observación diseñados para el caso. Esta variable no puede ser activa pues no es posible, para efectos de realizar una investigación, decidir que a un grupo de profesores se les asigne el entrenamiento integral y a otros, se les quite. Este es un intento de manipulación forzada que no funciona en este caso. Para estudiar las variables atributivas, el investigador debe proceder mediante procesos sistemáticos de observación. En cambio, las variables activas sí se manipulan. Una variable activa muy de actualidad es la modalidad de los estudios en la educación superior. En el año 2019, antes de la pandemia, el proceso educativo era presencial, así como en los demás niveles del sistema educativo peruano. Pero en el 2020, el proceso educativo ha cambiado, se ha manipulado, para hacerlo virtual. En cambio, en el 2021, muchos colegios particulares y hasta el Ministro de Educación están sugiriendo que las clases vuelvan nuevamente a ser presenciales. Este es un caso típico de manipulación de la variable modalidad de estudios y, en este caso, sí es posible hacer experimentos o cuasi experimentos. 
Por el método de estudio. Según este criterio, las variables son cuantitativas y cualitativas. Las variables son cuantitativas porque es posible medir sus magnitudes en una escala numérica. En cambio, las variables cualitativas no tienen masa, son fenómenos no másicos, no tienen magnitud, razón por la que es imposible medirlas. Pero para efectos de mejorar el análisis, el tratamiento de los datos o la discusión de los resultados, algunos investigadores convierten sus variables cuantitativas en cualitativas. Esto se llama degradación de la variable. En cambio, una variable cualitativa no puede convertirse en cuantitativa $\mathrm{y}$, si esto fuera posible, la variable no habría sido cualitativa sino, en esencia, era cuantitativa. Por ejemplo, la edad. Se puede decir que Pedro tiene 75 años, estimación cuantitativa, pero también se puede decir que Pedro es de la tercera edad o del grupo vulnerable de la población, estimación cualitativa. En definitiva, la edad es variable cuantitativa porque una persona vulnerable tiene 75 años, pero por alguna razón fue expresada cualitativamente.

Por los valores que adquieren. Según este criterio, las variables son dicotómicas y politómicas. Las variables dicotómicas son las que asumen sólo dos valores, como día y noche, vivo o muerto. Son pocas las variables realmente dicotómicas. La mayoría son politómicas, porque asumen tres o más valores. La variable condición socio económica se expresa en baja, media y alta, tres valores; pero también puede expresarse en cinco valores: muy baja, baja, intermedia, alta y muy alta. Las variables politómicas, para efectos del mejor tratamiento de los datos, o la discusión más precisa de los resultados, pueden convertirse en dicotómicas. Pero las dicotómicas no pueden convertirse en politómicas.
Y si esto ocurriera, la variable no habría sido dicotómica sino una auténtica politomía. Es muy conveniente convertir las politómicas en dicotómicas, porque ayuda a diseñar la mejor estrategia para probar la hipótesis. Con la variable condición socio económica se puede establecer una dicotomía: pobres y ricos, pero no por ello la condición social sea dicotómica.

Por la posesión de la característica. Según este criterio, las variables son categóricas, discretas y continuas. Las variables categóricas presentan una solución de continuidad entre cada una de sus variaciones, como en el estado civil: soltero, casado, conviviente, divorciado, viudo. En este caso, el soltero es soltero definitivamente y el casado lo es en el mismo sentido. No puede ser que haya una continuidad, aun sea muy tenue, entre ser soltero y estar casi casado. Las variables discretas son muy similares a las categóricas, pero son cuantitativas, como por ejemplo el número de quejas que presentan los usuarios o el número de proyectos de inversión ejecutados por una empresa. Estas variables, de ninguna manera, se pueden convertir en continuas. Y si así fuese, la variable habría sido una auténtica variable continua. Las variables continuas, en cambio, no presentan la solución de continuidad entre cada una de sus niveles de variación. Entonces queda la duda para muchos investigadores acerca de dónde radica la diferencia entre uno y otro nivel de variación. La diferencia radica en la cantidad de magnitud de la variable que hay en cada una de ellas. Pero como las diferencias son mínimas entre uno y otro nivel de variación, se dice que son continuas, como la inteligencia, pues a nadie se le puede ocurrir que una persona no tenga inteligencia y otra sí. La diferencia radica en que una persona tiene menos inteligencia que otra $y$, si esto es 
así, la variable, que también es cuantitativa, se puede medir en la escala que usan los psicólogos, o como la medición de las longitudes con el metro, o se puede hacer una escala especial, por ejemplo, para el dolor a partir de analizar que alguien diga, en una supuesta escala del dolor del 1 al 10, cuanto le duele algo en dicha escala. Esta escala se puede dividir en múltiplos y sub múltiplos para distinguir sutiles matices de variación. Las variables categóricas, por lo general, son cualitativas, mientras que las continuas son cuantitativas. Éstas últimas, para un mejor análisis de los datos o discusión de los resultados, pueden convertirse en categóricas, como aprobado o desaprobado, pese a ser una variable continua.

\section{Investigaciones bivariadas}

Tipificadas las investigaciones y clasificadas las variables según los criterios que, para ambos casos, queda explicado, el investigador ya posee elementos de juicio suficientes para saber con cuántas variables independientes va a trabajar y cuál es la variable dependiente de su estudio. Esto le permite diseñar, con mayor eficiencia, la estrategia para probar sus hipótesis y, lo que es más importante, elegir la prueba de hipótesis adecuada a los datos que obtendrá. El investigador no puede, arbitrariamente, elegir la prueba que desea aplicar, sin tener en cuenta los datos que ha obtenido.

Si el investigador tiene una variable independiente y otra dependiente, su investigación es bivariada. En la mayoría de los casos los estudiantes de posgrado realizan este tipo de investigaciones. Ellos elijen una variable independiente, llamada X y plantean la relación en el sentido que ésta es la causa de la otra variable, Y. En este caso, cuando el investigador tiene sólo dos variables, una indepen- diente y otra dependiente, no es posible ni necesario deducir sub hipótesis. En caso que así se haga, esto resulta totalmente contrario a la razón, pues lo mismo se afirma en la hipótesis general que en las supuestas sub hipótesis.

Para obtener estas supuestas sub hipótesis, los graduandos dividen la variable independiente, en sus dimensiones y elaboran tantas sub hipótesis como dimensiones hayan identificado en la variable independiente. Algunos estudiantes, en lugar de dividir la variable independiente, también dividen la dependiente en sus dimensiones constitutivas y así elaboran un número no justificado de sub hipótesis, en función del número de dimensiones identificadas en ambas variables. En el ciento por ciento de casos revisados, la conclusión general, es la sumatoria de las sub hipótesis, es decir, terminan llegando al lugar donde empezaron: $\mathrm{X}$ influye en $\mathrm{Y}$.

Con respecto a esta equivocada práctica de confundir las variables con pizzas, trataremos de explicar por qué no es posible generar sub hipótesis cuando el investigador tiene solo dos variables: una independiente, $\mathrm{X}$, y otra dependiente, Y. En algunos casos, los graduandos realizan investigaciones correlacionales y aun en estos casos, cuando correlacionan una variable con otra, redactan tantas sub hipótesis como dimensiones hayan identificado de las variables, lo que es inaceptable.

Los graduandos abusan de la formulación de sub hipótesis. Éstas se postulan sólo cuando se trabaja con dos o más variables independientes, como es el caso de algunas investigaciones factoriales. Por ejemplo, la calidad del servicio educativo está en función de la capacitación de docentes y se formaliza de 
del siguiente modo: $\mathrm{Y}=\mathrm{f}(\mathrm{X})$, ' $\mathrm{Y}$ está en función de X'. Como sólo hay dos variables, la investigación es bivariada. En este caso no caben sub hipótesis. Se trabaja con una sola hipótesis, que también se llama hipótesis general, hipótesis de trabajo, hipótesis alterna $y$, una vez formalizada, es la hipótesis estadística. Para probarla, se procede mediante la prueba inversa, motivo por el cual se debe formular la hipótesis nula: Y no es función de X.

Muchos graduandos elaboran una matriz de problematización, un 'árbol de problemas' y poco falta para que elaboren el 'bosque de problemas'. También grafican una 'espina de pescado', supuestamente para jerarquizar los problemas. Estas especificaciones son poco pertinentes. Si el graduando ya tiene serias dificultades para elaborar el problema central de la investigación, como en la mayoría de los casos, le resultará muy complicado plantear sub problemas o problemas específicos y presentar un 'árbol de problemas' o lo que últimamente se está llamando 'matriz de problematización'.

\section{Ejemplo de sub hipótesis tipo pizza}

En el siguiente ejemplo que, por razones éticas, mantenemos al autor en el anonimato, pero aclaramos que se han hecho algunos arreglos formales para mejorar la calidad del ejemplo, las variables son dos: variable independiente: estrategias de aprendizaje y variable dependiente: rendimiento académico en la asignatura de economía de la Facultad de Ciencias Administrativas de la UNMSM. En este caso, el graduando ha procedido del siguiente modo:

Para la variable independiente ha identificado las siguientes dimensiones: a) Dimensión cognitiva, b) Dimensión social, c) Dimensión indagación y, d) Dimensión tratamiento de la información. Según estas dimensiones, ha redactado el siguiente problema general:

\section{Problema general}

¿Cuál es la influencia de la variable estrategias de aprendizaje en el rendimiento académico en la asignatura de Economía de estudiantes de la Facultad de Ciencias Administrativas de la UNMSM, en el año 2017? Siguiendo con la tendencia de dividir la variable independiente en sus dimensiones constitutivas, para obtener los problemas específicos, ha procedido del siguiente modo:

\section{Problemas específicos}

- ¿Cuál es la influencia de la dimensión cognitiva de la variable estrategias de aprendizaje, en el rendimiento académico en la asignatura de Economía de estudiantes de la Facultad de Ciencias Administrativas de la UNMSM, en el año 2017? - ¿Cuál es la influencia de la dimensión social de la variable estrategias de aprendizaje, en el rendimiento académico en la asignatura de Economía de estudiantes de la Facultad de Ciencias Administrativas de la UNMSM, en el año 2017? - ¿Cuál es la influencia de la dimensión indagación de la variable estrategias de aprendizaje en el rendimiento académico en la asignatura de Economía de estudiantes de la Facultad de Ciencias Administrativas de la UNMSM, en el año 2017? - ¿Cuál es la influencia de la dimensión tratamiento de la información de la variable estrategias de aprendizaje, en el rendimiento académico en la asignatura de Economía de estudiantes de la Facultad de Ciencias Administra- 
tivas de la UNMSM, en el año 2017?

\section{Objetivo general}

Siguiendo la tendencia de dividir lo indivisible, para redactar los objetivos, el graduando ha procedido del siguiente modo: Establecer la influencia de la variable estrategias de aprendizaje, en el rendimiento académico en la asignatura de Economía de estudiantes de la Facultad de Ciencias Administrativas de la UNMSM, en el año 2017.

\section{Objetivos específicos}

- Establecer la influencia de la dimensión cognitiva de la variable estrategias de aprendizaje, en el rendimiento académico en la asignatura de Economía de estudiantes de la Facultad de Ciencias Administrativas de la UNMSM, en el año 2017. - Establecer la influencia de la dimensión social de la variable estrategias de aprendizaje, en el rendimiento académico en la asignatura de Economía de estudiantes de la Facultad de Ciencias Administrativas de la UNMSM, en el año 2017.

- Establecer la influencia de la dimensión indagación de la variable estrategias de aprendizaje, en el rendimiento académico en la asignatura de Economía de estudiantes de la Facultad de Ciencias Administrativas de la UNMSM, en el año 2017. - Establecer la influencia de la dimensión tratamiento de la información de la variable estrategias de aprendizaje, en el rendimiento académico en la asignatura de Economía de estudiantes de la Facultad de Ciencias Administrativas de la UNMSM, en el año 2017.

\section{Hipótesis general}

Existe una influencia directa y significativa de la variable estrategias de aprendizaje, en el rendimiento académico en la asignatura de Economía de estudiantes de la Facultad de Ciencias Administrativas de la UNMSM, en el año 2017.

\section{Hipótesis específicas}

Luego de confundir las variables con pizzas, ha elaborado las siguientes sub hipótesis.

- Existe influencia directa y significativa de la dimensión cognitiva de la variable estrategias de aprendizaje, en el rendimiento académico en la asignatura de Economía de estudiantes de la Facultad de Ciencias Administrativas de la UNMSM, en el año 2017. - Existe influencia directa y significativa de la dimensión social de la variable estrategias de aprendizaje, en el rendimiento académico en la asignatura de Economía de estudiantes de la Facultad de Ciencias Administrativas de la UNMSM, en el año 2017. - Existe influencia directa y significativa de la dimensión indagación de la variable estrategias de aprendizaje, en el rendimiento académico en la asignatura de Economía de estudiantes de la Facultad de Ciencias Administrativas de la UNMSM, en el año 2017. - Existe influencia directa y significativa de la dimensión tratamiento de la información de la variable estrategias de aprendizaje, en el rendimiento académico en la asignatura de Economía de estudiantes de la Facultad de Ciencias Administrativas de la UNMSM, en el año 2017. 


\section{Investigaciones multivariadas con sub hi- pótesis}

Cuando se trata de este tipo de investigaciones, el investigador debe trabajar con dos o más variables independientes y una dependiente. Esta hipótesis se formaliza como una función matemática del siguiente modo: $Y=f(X 1, X 2, X 3, \ldots X n)$

Como en el ejemplo hay más de dos variables independientes, la investigación se denomina multivariada o factorial. En este caso, la hipótesis anterior puede dividirse en las tres sub hipótesis siguientes: Sub hipótesis 1: $Y=$ $f(X 1)$, donde el factor X1 genera esta sub hipótesis. Sub hipótesis 2: $Y=f(X 2)$, donde el factor X2 genera esta sub hipótesis. Sub hipótesis 3: $Y=f(X 3)$, donde el factor $\mathrm{X} 3$ genera esta sub hipótesis. Se puede formular tantas sub hipótesis como variables independientes tenga identificadas el investigador. Sin embargo, es común hallar algunos errores de formalización. El primer error consiste en alterar la nomenclatura de la matemática $\mathrm{y}$ formular que $\mathrm{X}=\mathrm{f}(\mathrm{Y} 1, \mathrm{Y} 2, \mathrm{Y} 3, \ldots \mathrm{Yn})$, es decir que $\mathrm{X}$ está en función de varias $\mathrm{Y}$. El segundo error es dividir $\mathrm{Y}$ en sus dimensiones y así formular que $\mathrm{Y} 1, \mathrm{Y} 2, \mathrm{Y} 3, \ldots \mathrm{Yn}=\mathrm{f}$ $(\mathrm{X})$, es decir que varias $\mathrm{Y}$ están en función de $\mathrm{X}$, lo que equivale a pretender realizar tantas investigaciones como el número de dimensiones identificadas de la variable dependiente.

El siguiente problema científico es un ejemplo de investigación multivariada o factorial:

¿Qué efectos producen, la calidad del desempeño docente, (X1), y los métodos didácticos que se aplican, (X2), en la calidad de los aprendizajes de estudiantes de posgrado de la Facultad de Educación de la UNMSM, (Y)?

\section{Hipótesis general}

La hipótesis general de la presente investigación es la siguiente:

El eficiente desempeño docente, (A2), y la aplicación de métodos didácticos centrados en el aprendizaje, (B2), son factores que contribuyen a incrementar los niveles de aprendizaje de estudiantes de posgrado de la Facultad de Educación de la UNMSM, (Y).

\section{Hipótesis nula}

El aprendizaje de los estudiantes de posgrado dela Facultadde Educación de la UNMSM, (Y), no se incrementa ni con el eficiente desempeño docente, (A2), ni con la aplicación de métodos didácticos centrados en el aprendizaje, (B2). Nótese que, por exigencias de la aplicación del Análisis de Varianza, ANOVA, adecuado para este caso, el modelo estadístico obliga a cambiar la denominación de las variables X1 y X2 por A y B, respectivamente.

Como en esta hipótesis aparecen dos variables independientes: Desempeño docente, (A), ymétodos didácticos, $(B)$, es posible, en este caso, formular dos sub hipótesis a partir de cada una de las variables independientes. Pero previamente se hace variar cada una de las variables independientes, del siguiente modo: variable desempeño docente, (A), que varía en desempeño docente eficiente, (A2), y desempeño docente no eficiente, (A1); variable métodos didácticos, (B), que varía en métodos didácticos centrados en el aprendizaje, (B2), y métodos didácticos centrados en la enseñanza, (B1).

Para probar estas hipótesis, por el método hipotético deductivo, el investigador redacta hipótesis nulas, llamadas también hipótesis absurdas, con respecto a las cuales se adoptarán las decisiones y se intentará 
descartarlas en el proceso de prueba de hipótesis. En este caso, el sistema de hipótesis que se genera para esta investigación es el siguiente:

\section{Primera sub hipótesis alterna}

Se observa un incremento significativo en el aprendizaje del grupo de estudiantes de posgrado de la Facultad de Educación, (Y), que tuvo profesores con eficiente desempeño docente, (A2), con respecto al grupo de estudiantes que tuvo profesores con desempeño docente no eficiente, (A1). En esta sub hipótesis, el investigador estudia el efecto de la variable desempeño docente.

\section{Primera sub hipótesis nula}

No se observan diferencias significativas en el aprendizaje del grupo de estudiantes de posgrado de la Facultad de Educación, (Y), que tuvo profesores con eficiente desempeño docente, (A2), con respecto al grupo de estudiantes que tuvo profesores con desempeño docente no eficiente, (A1).

\section{Segunda sub hipótesis alterna}

Se observa un incremento significativo en el aprendizaje del grupo de estudiantes de posgrado de la Facultad de Educación, (Y), al que se aplicó métodos didácticos centrados en el aprendizaje, (B2), con respecto al grupo de estudiantes al que se aplicó métodos didácticos centrados en la enseñanza, (B1). En esta sub hipótesis, el investigador estudia el efecto de la variable métodos didácticos.

\section{Segunda sub hipótesis nula}

No se observan diferencias significativas en el aprendizaje del grupo de estudiantes de posgrado de la Facultad de Educación, (Y), al que se aplicó métodos didácticos centrados en el aprendizaje, (B2), con respecto al grupo de estudiantes al que se aplicó métodos didácticos centrados en la enseñanza, (B1).

\section{Tercera sub hipótesis alterna}

Previamente, para probar esta hipótesis, el investigador ya ha elegido la estrategia que aplicará y la prueba de hipótesis respectiva, es así como se explica que en este caso aplicará el Análisis de Varianza. Pero como para aplicar una prueba estadística previamente se debe establecer la normalidad de las muestras y establecer si existe covarianza entre las dos variables independientes se postula, por cuestiones previsionales, la hipótesis de la interacción de los factores en la variable dependiente.

Esta es una tercera hipótesis que surge de la posible interacción de los factores A y B, la que en términosliteralesseformula del siguientemodo: La interacción de los factores desempeño docente $y$ métodos didácticos, (A $x$ $B)$, incrementa significativamente los $n i$ veles de aprendizaje de estudiantes de posgrado de la Facultad de Educación.

\section{Tercera sub hipótesis nula}

La interacción de los factores desempeño docente y métodos didácticos, ( $\left.A \begin{array}{lll}x & B\end{array}\right)$, no produce efectos significativos en los niveles de aprendizaje de estudiantes de posgrado de la Facultad de Educación, (Y).

\section{Investigaciones multivariadas sin sub hipó-} tesis

Las investigaciones que, en los últimos años están realizando los estudiantes de maestría y doctorado, en casi todas las universidades del país, se hacen indiscriminadamente con sub hipótesis, a partir de la descomposición de una variable en sus dimensiones. Así, si para una variable identifican siete componentes, los graduandos elaboran siete sub hipótesis, haciendo corres- 
ponder cada dimensión con una sub hipótesis.

Otros estudiantes descomponen la variable independiente y la variable dependiente, en sus respectivas dimensiones y así elaboran una larga lista de sub hipótesis que corresponden a cada una de las dimensiones de las mencionadas variables.

Algunos graduandos dividen la variable dependiente en sus respectivas dimensiones y así, las variables independientes resultan estar en función de la dependiente, lo que es un absurdo matemático.

Al parecer el problema ha surgido de la confusión entre la operacionalización de las variables y la formulación de hipótesis. Cuando se operacionalizan las variables, es correcto identificar las dimensiones, factores, componentes, categorías, elementos, o aspectos. Existe una larga lista de sinónimos para esta idea que resulta innecesario crear, como muchos lo están haciendo, el nuevo sinónimo: 'sub variable’ que incrementa aún más la confusión metodológica, innecesariamente generada.

En algunas universidades, en los documentos orientadores para la realización de tesis se recomienda operacionalizar todas las variables de la investigación en un solo cuadro, como se observa en la Guía para el procedimiento de la elaboración de la tesis para la obtención del grado de magíster y doctor. (UPG-FE, 2020, p. 97), sin tener en cuenta que cada variable es una identidad y debe ser tratada como tal y operacionalizada por separado de las otras variables.

Toda variable es un constructo teórico, elaborado al más alto nivel de abstracción para referirse con ella a los fenómenos que estudian los científicos. (Mejía, 2017). Sin embargo, el con- cepto de constructo no se tiene en cuenta y, por el contrario, se recomienda que todas las variables de la investigación sean operacionalizadas en el mismo cuadro como si se tratara de un único concepto. Pero, cada variable, para ser operacionalizada, debe ser analizada en sus respectivas dimensiones, para elaborar el respectivo instrumento de acopio de datos.

Sin embargo, se ha generalizado la rutina de elaborar sub hipótesis a partir de las dimensiones de alguna variable. Aquí radica la confusión: redactar sub hipótesis a partir de la división de las variables en sus dimensiones constitutivas, sin advertir que cada variable es una entidad indivisible.

Pero aun cuando el investigador tuviera más de dos variables independientes y si entre éstas existiera covarianza, no es posible dividir esta hipótesis en tantas sub hipótesis como variables independientes se estudien. El siguiente es un ejemplo de una hipótesis que no genera sub hipótesis:

El índice académico, (X1), la organización del tiempo libre, (X2), y la afinidad de la actividad laboral con los estudios, (X3), son factores que influyen en el éxito académico de estudiantes de posgrado, (Y).

Esta hipótesis no se puede dividir en tres o cuatro sub hipótesis, porque entre las tres variables independientes existe covarianza, entonces, al no poder dividirse en sub hipótesis, la metodología recomienda aplicar el Análisis de Regresión Múltiple, que es una especie de licuadora en la que los datos de las variables independientes, como ya están en covarianza entre sí, se están entremezclando, resulta mejor terminar de mezclarlos totalmente para analizar la mejor combinación de estas va- 
riables y de este modo obtener puntuaciones predichas de Y. Luego se aplica la Prueba F para la Regresión Múltiple, (R), correlacionándolas con las puntuaciones reales de $\mathrm{Y}$. Este coeficiente explica qué porcentaje de Y es explicado por las variables independientes, sin necesidad de redactar sub hipótesis. Otra razón para no hacerlo es que las tres variables independientes atañen al estudiante y la dependiente, Y, también atañe al mismo estudiante, de modo que todas las variables, en este ejemplo, atañen al estudiante y así, todas se vinculan con todas. Es imposible graficar este fenómeno en el plano bidimensional del papel, porque se trata de relaciones volumétricas.

\section{CONCLUSIONES}

1.La formalización matemática reconoce a $\mathrm{X}$ como la variable independiente y a Y como la variable dependiente. Así, la formalización de una hipótesis bivariada es la siguiente: $Y=f(X)$; y la formalización de una hipótesis multivariada es: $Y=f(X 1, X 2, X 3, X n)$. 2.Cuando se investiga con dos variables, una independiente y otra dependiente, en el caso de las investigaciones explicativas, o se trata de asociar dos variables, en el caso de las investigaciones relacionales no causales, no es posible formular sub hipótesis, a partir de la descomposición de la variable independiente en sus dimensiones constitutivas. 3.Para generar sub hipótesis no se descompone la variable independiente y la dependiente en sus componentes constitutivos, ni mucho menos la variable dependiente, porque se trata del efecto. 4.En las investigaciones multivariadas o factoriales, cuando se trabaja con dos o más variables independientes y una dependiente, es posible for- mular sub hipótesis activando una de las variables para cada sub hipótesis. Pero esta práctica sólo se realiza si tales variables independientes no se hallan en covarianza entre ellas y se ha decido aplicar el Análisis de Varianza. 5.En las investigaciones multivariadas o factoriales, cuando existe covarianza entre las variables independientes, no se formulan sub hipótesis, pues los efectos de cada una de las variables, concurrente y concomitantemente, producen los efectos que se están estudiando. En este caso, se aplica el Análisis de Regresión Múltiple, estrategia que analiza la mejor combinación de las variables independientes en la dependiente.

\section{REFERENCIAS BIBLIOGRÁFICAS}

Ascarza, Lucero. (2021). Instituto Nacional de Salud: juez y parte en el caso 'Vacunagate'. 20 de marzo de 2021 de: https://saludconlupa.com/noticias/instituto-nacional-de-salud-juez-y-parte-en-el-vacunagate/

BBC NEWS/Mundo: Cómo Bill Gates se convirtió en el centro de tantas teorías de la conspiración en medio de la pandemia. 02 de marzo de 2021 de, https://www.bbc.com/mundo/ noticias-52952518

Bunge, M. (1983). La investigación cientifica. Ariel.

Hernández, Fernández y Baptista. (2009). Metodología de la investigación. Mc. Graw Hill.

Informe de la Comisión Sectorial Investigadora de la Aplicación de la Vacuna Candidata Contra la COVID-19. 1 de marzo del 2021 de: https://cdn.www.gob.pe/uploads/document/ file/1693259/INFORME\%20DE\%20LA\%20 
COMISION\%20SECTORIAL\%20INVESTIGADORA\%20DE\%20LA\%20APLICACION\%20DE\%20LA\%20VACUNA\%20 CANDIDATA $\% 20$ CONTRA $\% 20 \mathrm{LA} \% 20$ COVID\%20-\%2019\%20ok.pdf.pdf

Kerlinger, F, (2002). La investigación del comportamiento. 5 de enero de 2021 de: https:// fdocuments.ec/document/kerlinger-fred-n-investigacion-del-comportamiento.html

Klimovsky, G. (1994). Las desventuras del conocimiento científico. AZ Editora.

Mejía, E. (2017). La investigación cientifica en educación. Apeem, Editores.

Muy Interesante. (2021). Ocho frases de Galileo Galilei. 10 de marzo del 2021 de: https:// www.muyinteresante.es/cultura/arte-cultura/ articulo/ocho-frases-de-galileo-galilei\#: :text $=\% 22$ Mide $\% 2010 \% 20$ que $\% 20$ se $\% 20$ pueda,imposible $\% 20$ aprender $\% 20$ algo $\% 20$ de $\% 20$ ella.\%22

Piaget, J. (1979). Epistemología de las relaciones interdisciplinarias. 08 de febrero de 2021 de: https://www.semanticscholar.org/paper/La-epistemologia-de-las-relaciones-Piaget/ba90ecd441b737085c4d737e4fd6ca65a$6 \mathrm{c} 02 \mathrm{aa} 2$

Popper, K. (2017). La lógica de la investigación científica. Reimpresión de la $2^{\mathrm{a}}$. Edición. Tecnos.

Quacquarelli Symonds: Ranking de universidades del Perú. Recuperado el 03 de marzo de 2021 de. https://www.google. $\mathrm{com} /$ search $\mathrm{q}=$ ranking + de + universidades + del+Peru + seg $\%$ C3\%BAnQS\&oq=ranking+de+universidades + del + Peru + seg $\%$ C3\%BAn-
QS\&aqs=chrome..69i57j33i10i16014.13593j0j1$5 \&$ sourceid $=$ chrome $\&$ ie $=\mathrm{UTF}-8$

Quacquarelli Symonds: Ranking QS de las mejores universidades del mundo. 09 de marzo de 2021 de: https://www.revistanuve.com/ ranking-qs-mejores-universidades-del-mundo-2021/

UPG-FE/UNMSM. (2020). Guía para el procedimiento de la elaboración de la tesis para la obtención del grado de magíster y doctor. 